\title{
Identification of Voltage Pollution Source Based on Wavelet Transform in DC Power Supply System
}

\author{
Liu Dan ${ }^{1, a}$, Zhang Chenyu ${ }^{1}$, Li Qiang ${ }^{1}$, Yuan Xiaodong ${ }^{1}$, Wang Jian², Zhang \\ Xingsheng ${ }^{2}$, Miao Huiyu ${ }^{3, b}$ \\ ${ }^{1}$ Electric Power Research Institute, State Grid Jiangsu Electric Power Company, Nanjing China; \\ ${ }^{2}$ Nanjing Energy Digital Electric Co., Ltd., Nanjing, China; \\ ${ }^{3}$ College of Electric Engineering, Southeast University, Nanjing China; \\ a649711458@qq.com, bmhy034@163.com
}

\begin{abstract}
Keywords: Wavelet transform, DC power supply system, pollution identification
Abstract. The voltage problems in the DC voltage bus contains the voltage fluctuation, the voltage sag and the voltage interruption problems. In this paper, the wavelet module is established based on idea of wavelet transform. The voltage pollution source in the DC bus is identified by wavelet transform. In the Matlab simulation, different wavelet function can identify the fluctuation time of the DC voltage and the corresponding wave source, which has a good identification effect.
\end{abstract}

\section{Introduction}

The DC power supply system is a kind of system which is based on the interconnection of the DC bus, and there is no electric quantity such as frequency, phase and so on, compared with the AC power supply system. In the DC power supply system, the distributed power and energy storage devices are connected to the DC bus by the converter. DC power supplies such as solar cells and fuel cells, are connected to the DC bus directly, without any complex grid-connected converters. The input of power electronic devices is decreased with the less control difficulty and the cost of investment. With the great advantages compared with AC power supply system, DC power supply system is the important developing trend of power electronics.

The distributed power access to the DC power supply system is usually the uncertainty of distributed power supplies and converters, which can cause special electric energy quality problems in the system. Similar to the AC power supply system, the DC power supply system electrical energy quality is mainly affected by the power grid, connected distributed power supply, storage equipment, load characteristics and control strategy. The power quality problems can be solved from the source by the research on the main power quality phenomena in DC power supply system and the characteristics of the pollutant source. It has significant meanings to the application of DC power supply system, even to the replacement of the traditional AC distribution network.in the future.

Different from the AC distribution network, the power quality of the DC power supply system is mainly reflected in the fluctuation of the DC voltage. The stability of the DC voltage will directly affect the safety of the power grid. At present, for the transient electric quality detection problem, domestic and foreign scholars propose a variety of methods, including: the short-term Fourier transform, Hilbert Huang transform and dq transform method. There are still some disadvantages in these methods to identify the voltage fluctuation [1-3]. According to the existing public research data, the related research is still in initial stage.

The essence of the voltage pollution source identification in the DC power supply system is the detection of the singular point in the disturbing signal. Therefore, wavelet transform is applied in the paper, making full use of the characteristics of wavelet transform in the time frequency localization[4]. When electric quality problems in DC power supply system happens, there is a small mutation in the DC voltage waveform, which can be magnified by wavelet transform[5]. The electric quality problems can be positioned by detecting amplitude of the signal quality and the electric quality signal duration. The flow chart of voltage fluctuation source identification and location is shown in Fig.1. 


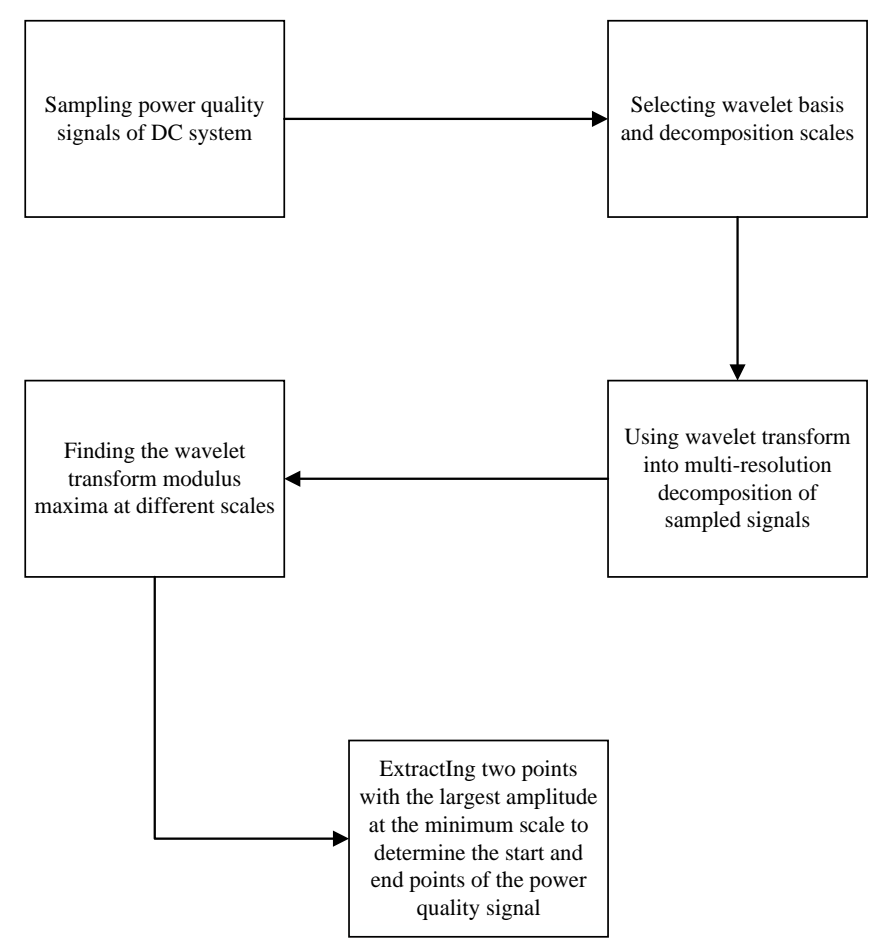

Fig. 1 The flow chart of voltage fluctuation source identification and location

\section{Main power quality problems in DC power supply system}

The main power quality of the DC power supply system is as follows[6]: voltage deviation, voltage fluctuation, voltage sag and voltage interruption, shown in Table 1.

Tab.1 Power quality problems in DC system

\begin{tabular}{|c|c|c|}
\hline power quality & Definition & Cause mechanism \\
\hline voltage deviation & $d(\%)=\frac{U_{\text {meas }}-U_{\text {rating }}}{U_{\text {rating }}} \times 100 \%$ & $\begin{array}{c}\text { Wire diameter, power supply distance, power flow } \\
\text { distribution, voltage regulation, load } \\
\text { characteristics }\end{array}$ \\
\hline voltage fluctuation & $d(\%)=\frac{U_{\max }-U_{\min }}{U_{N}} \times 100 \%$ & Fluctuation output power of distributed generation \\
\hline voltage sag & $\begin{array}{l}\text { The root mean square value } \\
\text { of voltage is reduced to } \\
0.1-0.9 \text { times the rated } \\
\text { voltage in power frequency } \\
\text { condition }\end{array}$ & System component or circuit fault, heavy load start \\
\hline voltage interruption & $\begin{array}{l}\text { The supply voltage is } \\
\text { reduced to } 0.1 \text { times the } \\
\text { rated voltage, and the } \\
\text { duration is not more than } 1 \\
\text { minutes. }\end{array}$ & $\begin{array}{l}\text { System failure, electrical equipment failure or } \\
\text { control failure }\end{array}$ \\
\hline
\end{tabular}

\section{Main idea of wavelet transform}

The main idea of wavelet transform is to represent or gain on the analyzed signal $f(t)$ by some function[7]. And the function is obtained by scaling and translation of the basic wavelet function $\Psi(t)$. The specific method is as follows.

Assume $\Psi(t) \in L^{2}(R)$, the Fourier transform is $\Psi(\omega)$, and $\Psi(\omega)$ meets the conditions as

$$
C_{\Psi}=\int \frac{|\Psi(\omega)|}{|\omega|}<\infty
$$


$\Psi(t)$ is called as basic wavelet function or generating wavelet function. Scale and translate it and the wavelet sequence is as

$$
\Psi_{a, b}(t)=\frac{1}{\sqrt{|a|}} \Psi\left(\frac{t-b}{a}\right)
$$

In formula (2), $a$ is the scaling parameter and $b$ is the dimension parameter. For any signal $f(t) \in L^{2}(R)$, it can be continuously wavelet transformed as

$$
W_{f}(a, b)=\frac{1}{\sqrt{|a|}} \int_{-\infty}^{+\infty} f(t) \Psi\left(\frac{\overline{x-b}}{a}\right) d x=f(x) \cdot \Psi_{a}(x)
$$

The reconstruction formula is as

$$
f(t)=\frac{1}{C_{\Psi}} \iint \frac{W_{f}(a, b)}{a^{2}} \Psi\left(\frac{t-b}{a}\right) d a d b
$$

The aim of location the voltage pollution source in the DC power supply system is to determine the starting and ending time of the disturbance[8]. As the disturbance moment usually corresponds to an abrupt change of the signal and signal change point is the wavelet transform modulus maximum value point, the disturbance moment can be determined by the wavelet transform modulus maximum points. The proof procedure is as follows:

Assume smooth low pass function is $\theta(x)$. Its integration is 1 , and it decays to 0 in the infinite distance. Its first derivative is as

$$
\phi(x)=\frac{d \theta}{d x}
$$

And $\phi(x)$ is a bandpass function, in accordance with the admissible conditions of wavelet

$$
\int_{-\infty}^{+\infty} \phi(x) d x=0
$$

As a result, $\phi(x)$ can be the wavelet transform generating function. Assume $\theta_{a}=\frac{1}{a} \theta\left(\frac{x}{a}\right)$ as $\theta(x)$ scaling to factor $a$. The wavelet function of scaling factor $a$ is

$$
\phi_{a}(x)=\frac{\phi\left(\frac{x}{a}\right)}{a}=\frac{d \theta\left(\frac{x}{a}\right)}{d x}=a \frac{d \theta_{a}\left(\frac{x}{a}\right)}{d x}
$$

According to formula (3), the wavelet transform of signal $f(x)$ in factor $a$ is

$$
W_{f}(a, x)=f(x) \cdot \phi_{a}(x)=f(x) \cdot\left(a \frac{d \theta_{a}(x)}{d x}\right)=a \frac{d\left[f(x) \cdot \theta_{a}(x)\right]}{d x}
$$

In fixed scale factor $a, W_{f}(a, x)$ is the first derivative of signal $f(x)$ through the smooth function $\theta_{a}(x)$. And the point of the modulus maximum value is the change local change point. So the wavelet transform modulus maxima point position can be used to track the change point of the signal.

Assume the high frequency coefficient of multi-resolution decomposition in wavelet transform is $C D_{n}$, the low frequency coefficient is $A_{n}, n$ is the number of factorization level. High frequency coefficient can response the details of the wavelet and the low frequency coefficient can response the general picture of the wavelet. The detail is as follows:

(1) Sampling. In order to satisfy the sampling theorem and the accuracy, the sampling frequency is set as $5 \mathrm{kHz}$, which means there are 5000 sample points in per cycle, and the sampling sequence is obtained.

(2) Selection of wavelet bases and decomposition scales. When the function is not continuous, it is called as singular function and the point is the singular point. The singular point of the signal can be detected by the wavelet transform modulus maxima. Db series wavelet function meets the orthogonality, time-frequency compactly support, and Mallat fast algorithm characteristics. The 
sampling sequence is decomposed to 5 levels by DB4 wavelet and the first and second level high-frequency coefficients are CD1 and CD2.

(3) Detection of the change point. The modulus maxima and the positions of CD1 and CD2 are obtained, which corresponds to the change point of the signal.

(4) Determining perturbation time. The moment corresponding to the modulus maxima is recorded, which corresponds to the time of the occurrence and the end of the transient disturbance. Time interval is the duration of disturbance.

(5) Identification of the types of transient disturbance. The signal can be decomposed by db4 wavelet transform and the low frequency wavelet coefficient is A5. The magnitude of the disturbance signal can be estimated by the amplitude information of A5, and then the type of transient power quality can be identified. The test result is relatively ideal.

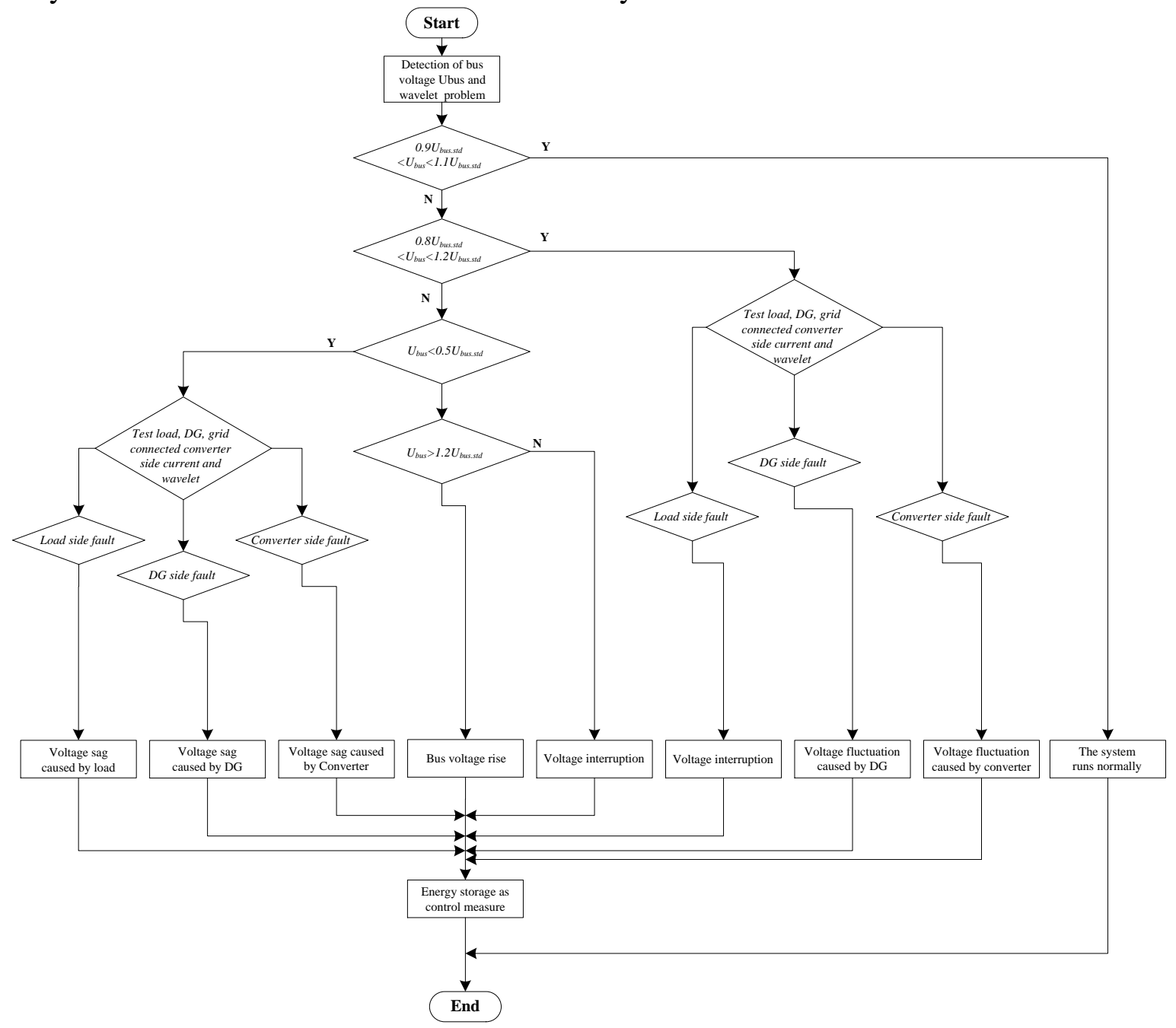

Fig. 2 The logic diagram of the DC bus voltage problem

Fig.2 shows the logic diagram of the DC bus voltage problem identification. The bus voltage problems are identified by the voltage variation range and wavelet transform. The wavelet transform detect the power quality problems when bus voltage changes. When the variation range is within $10 \%$, the change rate is used to identify the voltage deviation and voltage fluctuation. When the variation range is within $10 \%-90 \%$, it is assumed there is voltage sag in the sag. When voltage droops to below $10 \%$, the grid is identified as interruption. When the duration time is more than $3 \mathrm{~min}$, the interruption is considered as long time interruption. After the identification of power quality problems, wavelet decomposition in different modules detect the singularity to select the problem location module. Considering the voltage long time interruption, the power supply system is disconnected to the power grid and all the load is removed to reduce the scope of the accident. 


\section{Simulation verification}

In order to verify the identification of electric energy quality in DC power supply system based on $\mathrm{db} 4$ wavelet. The simulation is established in MATLAB/Simulink. There are PV and impact load in the DC power supply system. The rated DC voltage value is $380 \mathrm{~V}$. The horizontal and vertical coordinate in Fig.3-5 is time $t$ and voltage $U$.

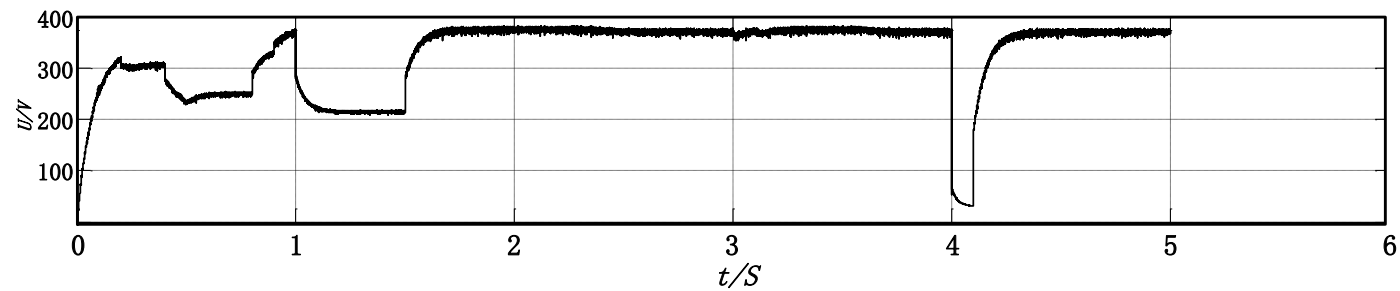

(a)

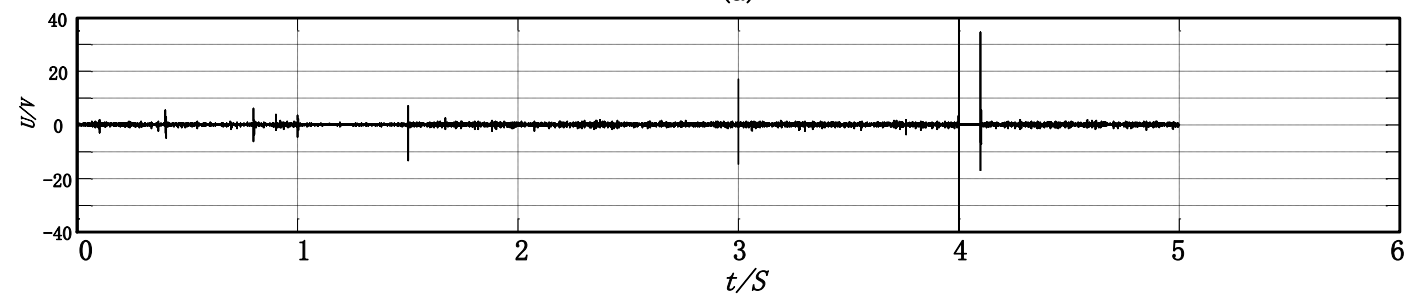

(b)

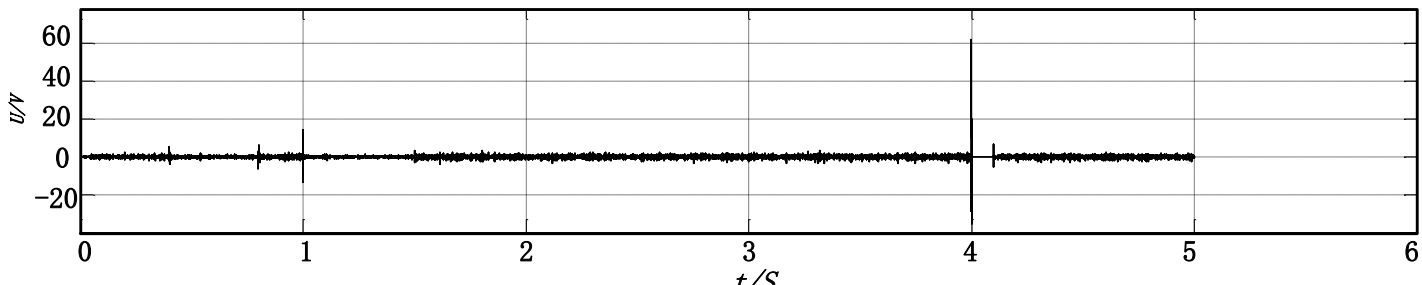

(c)

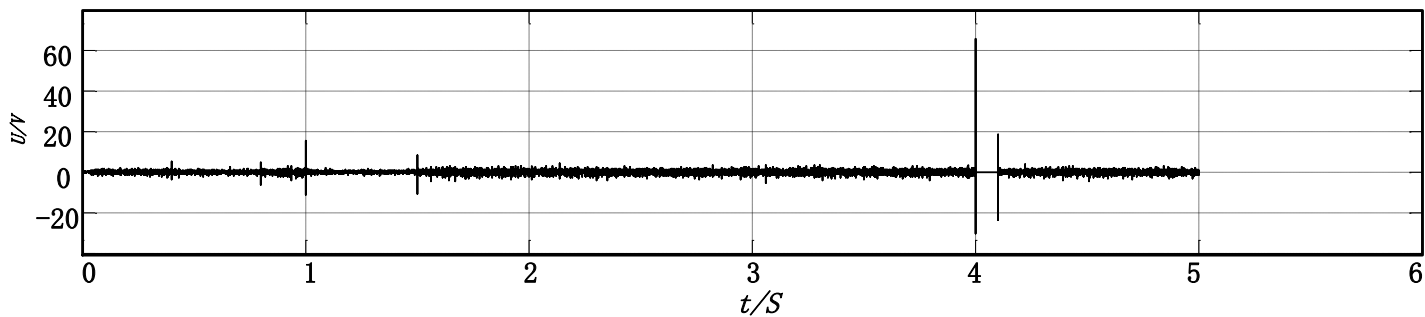

(d)

Fig.3 DC bus voltage analysis by db4, db 5, db6 wavelet

Fig. 3 shows the DC bus voltage problems detected by wavelet decomposition. Fig.3(a) shows the voltage in the DC bus. Fig.3(b), (c), (d) show the decomposition results by wavelet. db4 decomposition effect is the best. As shown in Fig.3, there are voltage fluctuation during 0-1s, voltage sag during $1-1.5 \mathrm{~s}$, voltage abnormity at $3 \mathrm{~s}$, voltage interruption at $4 \mathrm{~s}$. The voltage is normal in the other left time. All the electric quality problems are detected by db4 wavelet with singularity in the figure. 


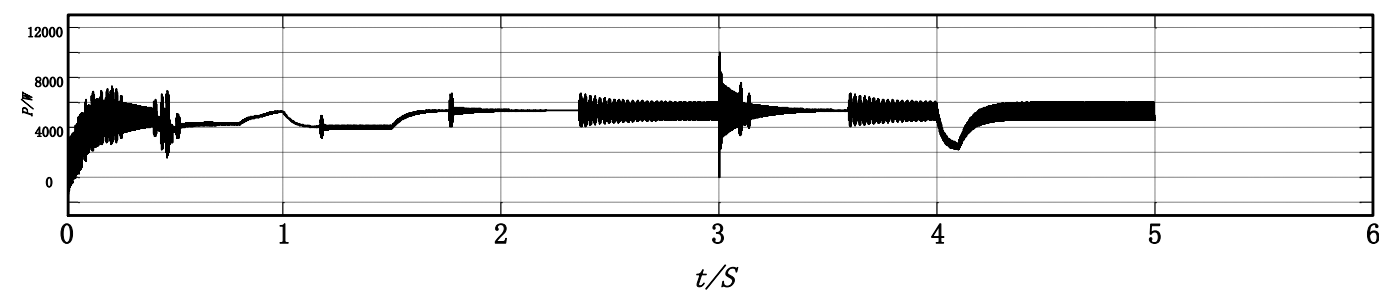

(a)

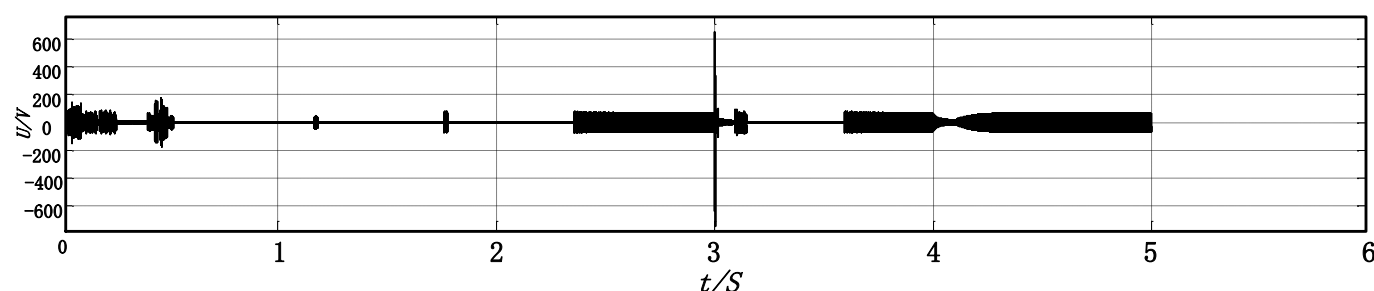

(b)

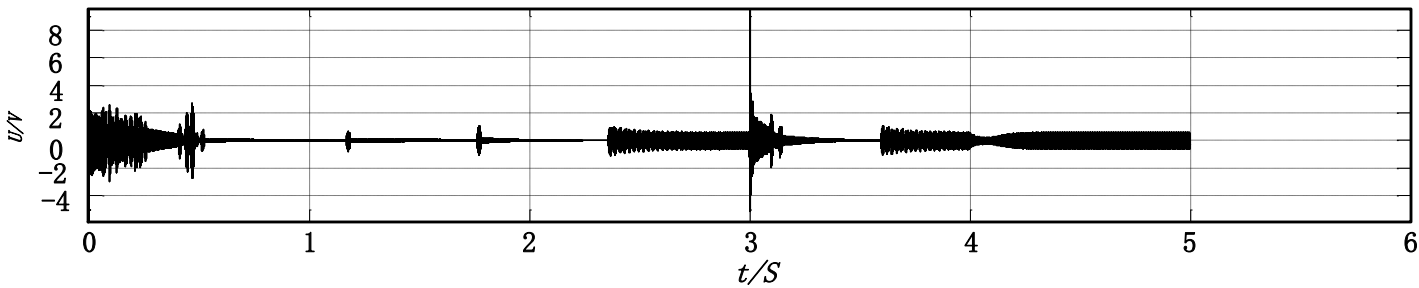

(c)

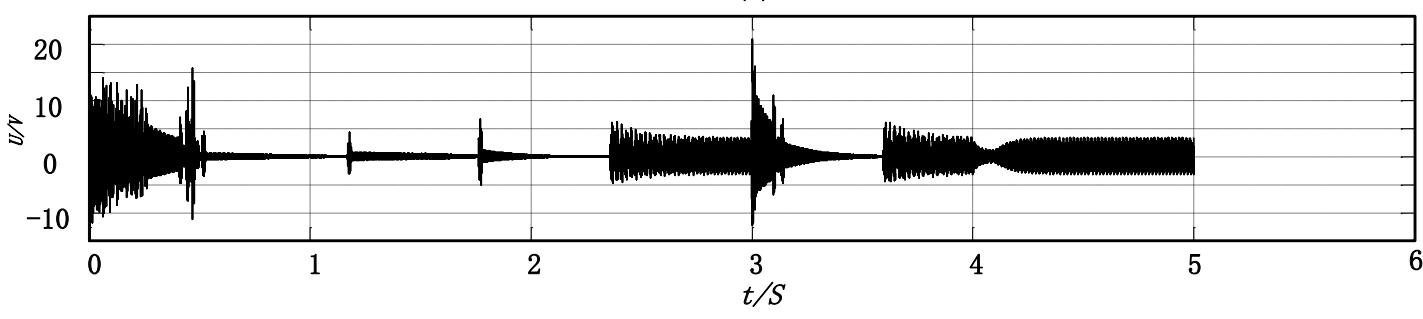

(d)

Fig.4 Output analysis of PV cells by db4, db5, db6 wavelet

Fig.4 shows the output power analysis of PV cells. In the simulation, there is an sudden change in the light radiation of PV cells at 3s. Fig.4(a) shows the output waveform of the PV cells. Fig.4(b), (c), (d) show the detection of the output by db6, db4, db5. And the decomposition result of db6 is the best. As shown in the figure, there is a sudden change in the light radiation at $3 \mathrm{~s}$, and the output of PV is abnormal which is detected by the wavelet transform. At other time, the output of PV is slightly affected by the other devices in the DC bus. The wavelet decomposition detect no power quality problems in PV. And the results are ideal.

Fig.5 shows the load power analysis by db4, db5, db6 wavelet. In the simulation, the load fluctuation causes the DC voltage fluctuation at $0-1 \mathrm{~s}$. There is voltage sag caused by load at 1-1.5s. And there is voltage interruption in the DC bus caused by the injection of impact load at 4s. Fig.5(a) shows changes of load power with fluctuation at 0-1s and impact at 4s. Fig.5(b), (c), (d) shows decomposition result by db6, db4, db5 wavelet. And the decomposition results of db6 is the best. There are singularities at $0.2 \mathrm{~s}, 0.4 \mathrm{~s}, 0.6 \mathrm{~s}, 0.8 \mathrm{~s}, 1 \mathrm{~s}, 1.5 \mathrm{~s}$ and $4 \mathrm{~s}$ which means the electric quality problems in the load side is detected. 


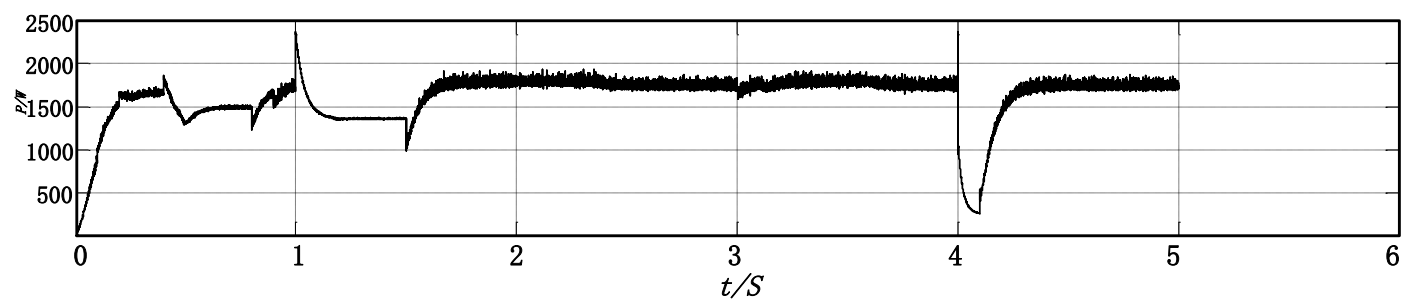

(a)

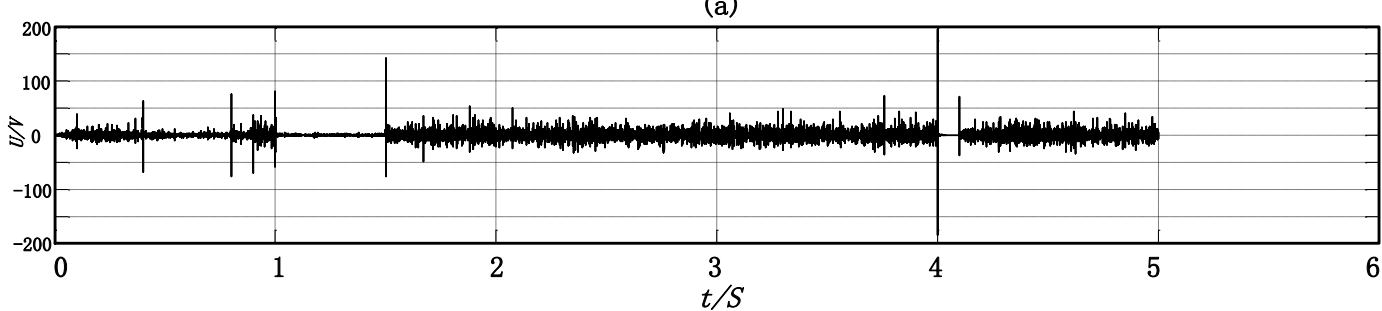

(b)

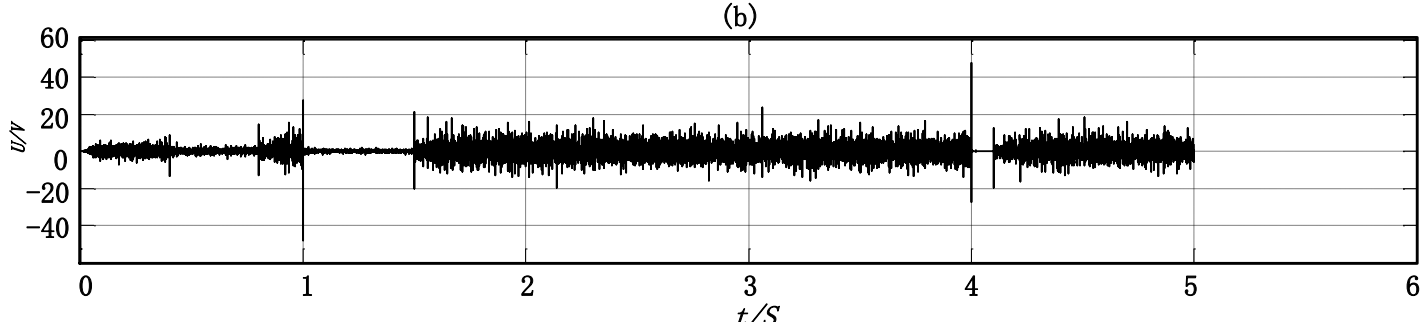

(c)

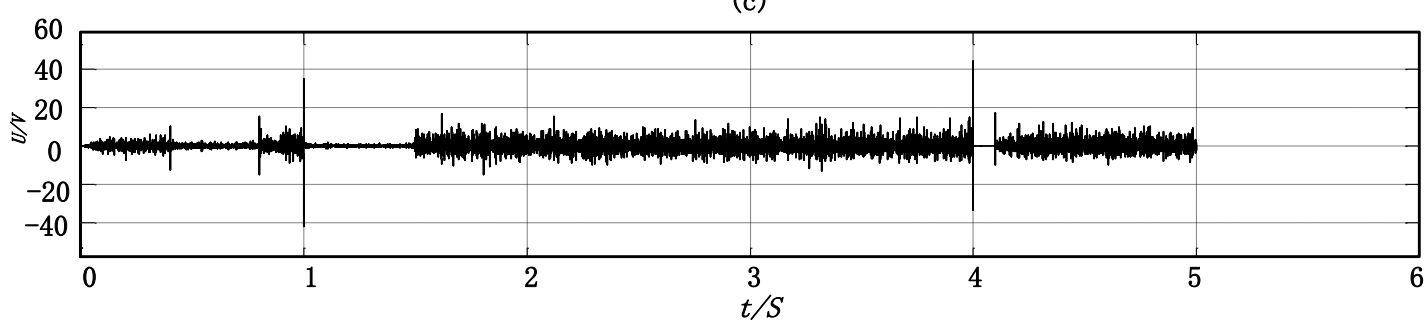

(d)

Fig.5 Load power analysis by db4, db5, db6 wavelet

\section{Conclusions}

The identification of voltage source in the DC system is introduced in the paper based on wavelet transform and the idea of wavelet transform is analyzed. Based on the simulation in the MATLAB, the wavelet analysis can identify the DC voltage fluctuation time and the corresponding pollution sources. However, its effectiveness still need to be verified with a large number of actual data.

\section{References}

[1] G. Wiczynski, "Voltage-Fluctuation-Based Identification of Noxious Loads in Power Network," in IEEE Transactions on Instrumentation and Measurement, vol. 58, no. 8, pp. 2893-2898, Aug. 2009.

[2] F. Xu, L. Shi, K. Wang, Z. Li, F. Gao and Y. Li, "A novel parameter identification of IM based on inverter by reducing dead-time effect," Power Electronics and Applications (EPE'15 ECCE-Europe), 2015 17th European Conference on, Geneva, 2015, pp. 1-9.

[3] G. Wiczynski, "Description of voltage fluctuations in LV power network with the use of Pst indicator and voltage fluctuation indices," 2008 13th International Conference on Harmonics and Quality of Power, Wollongong, NSW, 2008, pp. 1-4. 
[4] H. Gu, F. Zhang, Z. Wang, Q. Ning and S. Zhang, "Identification method for low-voltage Arc fault based on the loose combination of wavelet transformation and neural network," Power Engineering and Automation Conference (PEAM), 2012 IEEE, Wuhan, 2012, pp. 1-4.

[5] M. Wu, B. Xu, W. Cao and J. She, "Aperiodic Disturbance Rejection in Repetitive-Control Systems," in IEEE Transactions on Control Systems Technology, vol. 22, no. 3, pp. 1044-1051, May 2014.

[6] Zhang Chenyu, Zheng Jianyong, Mei Jun, et al. Control method for fault-tolerant active power filters[J]. Journal of Power Electronics, 2015, 15(3): 796-805.

[7] T. Y. Doh and J. R. Ryoo, "Robust approach to repetitive controller design for uncertain feedback control systems," in IET Control Theory \& Applications, vol. 7, no. 3, pp. 431-439, February 14 2013.

[8] I. Houtzager, J. W. van Wingerden and M. Verhaegen, "Rejection of Periodic Wind Disturbances on a Smart Rotor Test Section Using Lifted Repetitive Control," in IEEE Transactions on Control Systems Technology, vol. 21, no. 2, pp. 347-359, March 2013. 